\title{
Figures disruptives et cadres de l'expérience vidéoludique dans les JRPEV goréens : une approche sociocritique des jeux de rôle en ligne
}

\section{Christophe Duret}

\section{(2) OpenEdition \\ Journals}

Édition électronique

URL : http://journals.openedition.org/sdj/400

DOI : $10.4000 /$ sdj. 400

ISSN : 2269-2657

Éditeur

Laboratoire EXPERICE - Centre de Recherche Interuniversitaire Expérience Ressources Culturelles Education

\section{Référence électronique}

Christophe Duret, «Figures disruptives et cadres de l'expérience vidéoludique dans les JRPEV

goréens : une approche sociocritique des jeux de rôle en ligne », Sciences du jeu [En ligne], 3 | 2015, mis en ligne le 22 juillet 2015, consulté le 02 mai 2019. URL : http://journals.openedition.org/sdj/400 ;

DOI : $10.4000 /$ sdj. 400

Ce document a été généré automatiquement le 2 mai 2019.

Tous droits réservés 


\title{
Figures disruptives et cadres de l'expérience vidéoludique dans les JRPEV goréens : une approche sociocritique des jeux de rôle en ligne
}

\author{
Christophe Duret
}

\section{Introduction}

1 En 2012, les jeux vidéo ont généré des revenus de 78,5 milliards de dollars US, dont 24 milliards pour les jeux en ligne à eux seuls (Reuter, 2012). Déjà en 2008, les revenus liés à la vente de jeux sur PC et sur consoles rivalisaient avec ceux de l'industrie du cinéma à Hollywood (McCauley, 2008). Au regard de leur popularité et de leur importance au sein de l'industrie culturelle, les jeux vidéo constituent désormais des phénomènes médiatiques incontournables. En tant que tels, ils ont su attirer l'attention, au sein des game studies, de chercheurs soucieux d'y analyser les différentes forme d'inscription de la socialité, c'est-à-dire cette «forme socialisée du réel [...] [qui se réfère à] un espace, un temps, un être ensemble, un système de codes, un système de relations et d'interlocuteurs, un complexe de normes, d'idées » (Duchet et Maurus, 2011, p. 19). En considération des pans de la socialité abordés, il convient de mentionner la présence de tropismes qui ont pour conséquences de limiter son étendue aux stéréotypes raciaux et sexuels (voir, notamment, Higgin, 2009; Langer, 2008 ; Massie, 2011 et Monson, 2012), d'une part, et, d'autre part, aux discours idéologiques dominants, avec une insistance sur le colonialisme, l'impérialisme, le militarisme et le capitalisme (pensons, par exemple, à Cassar, 2009 ; Crogan, 2011 ; Dyer-Witheford et de Peuter, 2009 et Lizardi, 2009). Que ces études suivent les tropismes susmentionnés ou qu'elles s'intéressent à une autre dimension de la socialité, elles tendent à adopter une perspective axée sur l'objet au mépris d'une perspective axée sur le joueur, suivant la polarisation mise au jour par Juul 
(2010) dans les game studies ${ }^{1}$. Or, la circulation d'éléments de socialité est parfois indissociable de la dimension expérientielle du jeu, cette dernière concernant la seconde perspective. Dès lors, la lecture de l'objet vidéoludique nécessite la prise en considération de cette expérience, c'est-à-dire de la manière dont elle favorise la migration de la socialité entre les différentes composantes du jeu, entre sa diégèse et sa structure formelle, notamment. C'est ce qui se produit lorsqu'il y a disruption de l'expérience vidéoludique et que les figures disruptives qui en sont responsables, auxquelles nous consacrerons cet article, charrient avec elles des éléments de socialité incompatibles ou subversifs au regard du jeu dans lequel elles se manifestent.

2 Conséquemment, nous nous intéresserons aux figures disruptives émaillant les jeux de rôle goréens, soit ces figures qui, par leur brusque survenue, sont susceptibles de créer des contacts momentanés entre deux niveaux de signification du jeu normalement cloisonnés. Nous suivrons une démarche sociocritique instruite par l'analyse des cadres de l'expérience vidéoludique, soucieuse de faire converger une perspective centrée à la fois sur l'objet et le joueur.

3 Les jeux de rôle participatifs en environnement virtuel (ci-après dénommés «JRPEV ») ont lieu dans des environnements virtuels en ligne multi-usagers (MUVE) tels que Second Life et IMVU, au sein desquels les joueurs, suivant une logique participative, conçoivent les décors, les dispositifs techniques (compteurs de vie, scripts ${ }^{2}$, etc.), les règlements et les accessoires nécessaires à son fonctionnement. Les jeux de rôle goréens sont des jeux de rôle participatifs en environnement virtuel organisés dans Second Life. Ils s'inspirant des Chroniques de Gor (ci-après, les « Chroniques »), un cycle composé de 33 romans écrits entre 1966 et 2013 par John Norman. Les Chroniques mêlent science-fiction et culture antique et médiévale en suivant les conventions du genre de la romance planétaire ${ }^{3}$.

Les Chroniques sont sous-tendues par la loi de l'ordre naturel, une philosophie néodarwinienne qui se dessine à contrecourant des discours des mouvements féministes américains des années 1960. Selon cette thèse, et pour reprendre les mots de Norman, «le sexe masculin est naturellement dominant et le sexe féminin, réactif à la dominance » (cité dans Smith, 1996, s. p.). Les femmes sont donc, selon la loi de l'ordre naturel, biologiquement prédisposées à être soumises aux hommes et elles s'épanouissent dans cette relation de complémentarité des sexes. La loi de l'ordre naturel structure la planète Gor et ses différents peuples, dont l'organisation sociale est axée sur l'esclavage.

5 La communauté goréennes de Second Life regroupe deux types de joueurs caractérisés par leur posture interprétative sur les Chroniques. D'une part, on y retrouve les joueurs qui se veulent fidèles aux romans de Norman dans leur jeu de rôle. Ils se qualifient de By the Books (BtB). D'autre part, il y a les joueurs qui se distancient du contenu des romans pour introduire dans le jeu des éléments exogènes. Ce sont les joueurs Gor Evolved (GE). Les conflits entre les deux groupes concernent, pour l'essentiel, le rôle des femmes dans la société goréenne et la loi de l'ordre naturel. Les BtB affirment que dans le cadre de la planète Gor la femme n'est pas l'égale de l'homme. Les GE affirment au contraire qu'il est réaliste de concevoir que les Goréens aient évolué socialement au point de considérer la femme comme l'égale de l'homme.

6 En vue de décrire les figures disruptives telles qu'elles se présentent dans les jeux de rôle goréens, nous décrirons, dans la première section de cet article, l'expérience vidéoludique des JRPEV goréens en termes de cadres et de niveaux de signification, en nous appuyons sur Gary Alan Fine (1983) et Erving Goffman (1991). Dans la deuxième 
section, nous décrirons les principales caractéristiques des figures disruptives en termes expérientiels. Dans la troisième section, deux cas de disruption seront décrits à titre illustratif. Dans la dernière section, enfin, nous examinerons la portée sociocritique des figures disruptives et l'illustrerons à l'aide d'un troisième cas.

7 S'il constitue pour l'essentiel une proposition théorique, cet article repose néanmoins sur une analyse des documents produits par des membres de la communauté goréenne de Second Life qui témoignent, de manière indirecte, de leur pratique vidéoludique. Nous avons principalement travaillé à partir d'un corpus de près de 5000 billets répartis sur 70 fils de discussion, publiés sur les forums de discussion The Gorean Forums et Gor-SL. Nous avons également analysé des blogues de joueurs, des captures d'écran et des captures vidéo de séances de jeu ainsi que des encyclopédies en ligne et des revues amateur consacrés aux JRPEV goréens et aux Chroniques. Nous nous sommes, enfin, adonnés à de l'observation participante entre l'été 2012 et aujourd'hui.

\section{Les niveaux de signification dans les JRPEV ${ }^{4}$}

8 La question des figures disruptives soulève celle des niveaux de signification constitutifs de l'expérience vidéoludique. Nous décrirons donc les seconds avant de définir les premières.

9 Lorsqu'il se penche sur l'organisation de l'expérience dans les jeux de rôle sur table, Fine (1983) s'appuie sur la notion de cadres de Goffman (1991), ces schèmes interprétatifs qui ont pour fonction, lorsqu'ils sont mobilisés par un individu, de comprendre les événements, c'est-à-dire d'interpréter une situation dans laquelle il est impliqué ou dont il est témoin et être en mesure de répondre à la question «qu'est-ce qui se passe ici ?» Goffman distingue deux types de cadres, les cadres primaires et leurs modalisations. Parmi les cadres primaires, les cadres sociaux favorisent la compréhension d'événements pilotés par une intelligence. Ainsi, résume Goffman, grâce aux cadres sociaux, « [1]a vie sociale est organisée de telle sorte que nous pouvons généralement comprendre ce qui nous arrive et y prendre part » (p. 35).

10 Certains cadres font l'objet d'une modalisation, c'est-à-dire qu'en vertu de conventions, une activité normalement interprétée à l'aide d'un cadre primaire est transposée en une autre activité prenant cette dernière pour modèle. C'est le cas d'une pièce de théâtre, lorsqu'elle modalise des interactions sociales réelles telles qu'une réunion de famille, un conflit, une fête, un charivari, etc. Goffman distingue cinq modes fondamentaux, parmi lesquels se trouvent le faire-semblant (fantasmes, théâtre, etc.), mode qui concerne au premier chef les jeux de rôle.

11 Fine (1983) mobilise l'analyse des cadres afin de comprendre comment les rôlistes interprètent leur expérience de jeu et passent d'un monde social à l'autre. Dans une optique goffmanienne, les jeux de rôle se composent de trois niveaux interreliés de significations : 1) le cadre primaire, soit la compréhension de sens commun que les gens ont du monde réel, qui dépend des événements réels, 2) le cadre du jeu, ses règles et ses contraintes, et 3) le cadre de la fantaisie du jeu, au sein duquel les joueurs n'incarnent pas un personnage mais sont leur personnage. Les deux derniers niveaux constituent des modalisations de cadres primaires.

12 Nous inspirant de Fine, nous avons divisé les JRPEV en trois niveaux de signification constitutifs de l'expérience de jeu, soit le social, le ludique et le diégétique, régis tous 
trois par leur cadre respectif. Ces niveaux sont à distinguer des lieux concrets où se déroulent les JRPEV (forums de discussion, environnements virtuels, etc.), ces derniers peuvent être le théâtre de plusieurs niveaux, convoqués en alternance ou simultanément. Les niveaux de signification impliquent des lieux concrets, mais également des entités plus abstraites : la structure formelle et la diégèse du jeu. La première peut être définie, grossièrement, comme l'ensemble des règles, paramètres et dispositifs techniques qui encadre les actions des joueurs et simule le monde du jeu, lui confère cohérence et jouabilité (Dormans, 2006 ; Fine, 1983 ; Stenros et Hakkarainen, 2003). La seconde, quant à elle, désigne l'espace fictif à l'intérieur duquel évoluent les personnages des joueurs, dont ces derniers modifient l'état par leurs actions. Elle est régie par des lois et une cohérence distinctes du monde du hors-jeu (le monde « réel») (Fine, 1983; Gade, 2003; Hitchens et Drachen, 2009; Holter, 2007; Morgan, 2002; Padol, 1996). La structure formelle lui confère sa jouabilité et régit une part de ses lois et de sa cohérence. Le niveau social est subdivisé en quatre niveaux régis par leurs règles et leurs cadres propres. Les interactions sociales s'y déroulant relèvent du hors-jeu (niveau du hors-jeu) ou du jeu extrinsèque (niveaux méta-ludique, exégétique et herméneutique).

Suivant la distinction apportée par Siang Ang et al. (2010), nous opérons une distinction entre les formes intrinsèque et extrinsèque du jeu. Si le premier est contenu dans les limites prédéterminées de la structure du jeu, le second les déborde, constitué d'activités périphériques comme la rédaction de récits a posteriori ${ }^{5}$ ou des discussions visant à remettre en question, amender ou interpréter les règles du jeu.

Le niveau du hors-jeu renvoie aux interactions sociales qui, sans être en relation avec le jeu, ont lieu dans ses limites intrinsèques (dans les sims ${ }^{6}$ goréens) et extrinsèques (dans les forums et les blogues de joueurs, par exemple). Ce sont les interactions du joueur non engagé dans le jeu intrinsèque ou le jeu extrinsèque.

Le niveau méta-ludique est le théâtre d'interactions visant à réguler le jeu : conflits dans l'interprétation des règlements, modification ou création de nouveaux scripts ou accessoires, etc. C'est à partir de ce niveau et du niveau exégétique que les joueurs construisent collectivement les JRPEV goréens.

17 Le niveau exégétique émerge là où les joueurs s'adonnent à une activité interprétative portant sur les textes dont les JRPEV s'inspirent de manière privilégiée.

18 Le niveau herméneutique, quant à lui, se forme là où les joueurs s'adonnent à une activité réflexive au cours de laquelle leur interprétation des textes vidéoludiques - c'est-à-dire le fruit des interactions des personnages des joueurs entre eux et avec le monde du jeu simulé par la structure formelle - permet de porter un éclairage particulier sur leur vie quotidienne (hors-jeu) et sur le monde contemporain. Ce niveau rend visible la portée herméneutique du texte et sa pertinence pour l'ordonnancement des occurrences et expériences humaines discordantes tirées du continuum du réel en des totalités cohérentes, intelligibles (Ricœur, 1986), lequel ordonnancement favorise chez le joueur une compréhension de soi (et du monde) «médiatisée par des signes, des symboles et des textes » (Ricœur, 1986, p. 33).

19 Au niveau ludique ont lieu les interactions des joueurs avec la structure formelle et l'environnement du jeu (avec leurs règles, leurs objectifs et leurs obstacles) appréhendés dans une logique de compétition, de performance et d'optimisation ${ }^{7}$. Par exemple, si les dégâts engendrés par un coup d'épée sont interprétés, sur le plan diégétique, comme la conséquence sanglante d'une traitrise, ils le sont plutôt, au niveau ludique, comme la 
perte d'X points de vie. Le cadre ludique amène le joueur à considérer que les actions qu'il pose se situent entre parenthèses et relèvent du jeu (Bateson, 1977).

Le niveau diégétique est celui au sein duquel sont traduites les interactions des joueurs (entre eux et avec la structure formelle et l'environnement du jeu) en termes diégétiques. Elles sont intégrées au monde fictif du jeu. À ce niveau-là, les interactions ne sont plus attribuables aux joueurs, mais aux personnages qu'ils incarnent, selon le mode du fairesemblant. Par exemple, un joueur est susceptible d'interpréter diégétiquement l'intrusion, dans un sim goréen, de l'avatar d'un usager non-joueur vêtu à la mode punk comme une manifestation divine, alors qu'en termes méta-ludiques, il pourrait l'interpréter comme un faux pas commis par un néophyte qui maîtrise mal les règles de conduite de Second Life. Le cadre diégétique conduit le joueur à considérer les situations auxquelles il est confronté comme de la fiction.

\section{Figures disruptives}

21 Selon Eddo Stern (2002), il existe, dans les MMOG, une importante demande, auprès des joueurs technophiles, pour ce qu'il appelle des "privilèges cyberculturels » (accès à des espaces non-cartésiens, régénération sans fin du corps, etc.), de même que pour l'expérimentation d'une fantaisie dans un monde médiéval magique. Cyberculture et culture médiévale étant incompatibles, l'auteur se demande comment ces exigences peuvent cohabiter sans que les éléments de la première ne gâchent la fantaisie de la seconde. Il avance que cette coexistence est rendue non problématique en raison de la présence d'éléments de récits diégétiques (diegetic narrative elements), dont certains d'entre eux, qui nous intéressent ici, sont décrits comme des «artefacts métaphoriquement rapiécés » (metaphorically patched artifacts), des éléments de récits qui sont mis en place pour s'adapter à la diégèse par le déploiement d'une métaphore et qui ont pour but de dissimuler l'intrusion de dispositifs techniques dans la fantaisie du jeu. Par exemple, la possibilité technologique, pour un avatar, de voyager instantanément d'un point $\mathrm{A}$ à un point $\mathrm{B}$, distants l'un de l'autre, est dissimulée par la métaphore du portail magique. En utilisant de tels artefacts, on épargne au joueur le bris de ce que plusieurs chercheurs et joueurs appellent l'immersion. Or, il est des situations où ces intrusions ne sont pas diégétiquement camouflées, en particulier dans les JRPEV goréens, qui sont organisés dans un environnement virtuel non expressément défini pour le jeu. Il y a alors danger de disruption des formes ludique et diégétique de l'expérience des joueurs qui y sont confrontés. Nous désignerons sous le concept de figures disruptives de telles intrusions.

Comme l'observe Fine (1983), les joueurs, dans les jeux de rôle, tendent à osciller entre les différents niveaux de signification et ces oscillations, dans un environnement complexe comme celui des JRPEV goréens - où se croisent des usagers non-joueurs de Second Life, de simples observateurs venus assister aux jeux de rôle goréens, attirés qu'ils sont par leur réputation sulfureuse, des trouble-fête ${ }^{8}$, des rôlistes, des adeptes de BDSM, etc. - sont fréquents et généralement imprévus et incontrôlables. C'est ce qu'il se produit lorsque les niveaux ludique et diégétique sont soumis à la pression des figures disruptives. Les figures disruptives sont susceptibles de provoquer l'oscillation involontaire du joueur entre deux niveaux de signification du jeu en raison des ruptures de cadre qu'elles occasionnent, c'est-à-dire la dislocation de cadres sous la pression d'un facteur 
perturbateur, dislocation qui mène à l'impossibilité pour les acteurs sociaux impliqués de sauvegarder l'intégrité de leur rôle (Goffman, 1991).

Nous avons vu ailleurs (Duret, 2014a; suivant Calleja, 2011 et Thon, 2008) que ces oscillations et le degré d'implication des joueurs dans un niveau de signification donné (un haut degré d'implication étant généralement qualifié " d'immersion ", dans les game studies) s'expliquent en termes cognitifs de distribution des ressources attentionnelles. Le maintien et l'engagement profond et durable du joueur avec un niveau de signification du jeu est tributaire de sa capacité à lui allouer suffisamment de ressources attentionnelles, ceci, en vue d'éviter tout désengagement vis-à-vis de ce niveau (un bris d'immersion) ou sa contamination par d'autres niveaux concurrents. La rupture de cadre produite par la survenue de figures disruptives conduit à un désengagement involontaire du joueur d'une activité modalisée, provoqué par une incapacité (temporaire ou permanente) de mobiliser les ressources attentionnelles nécessaires à leur maintien. Ce faisant, le joueur délaisse le niveau de signification modalisé pour regagner le niveau social. Par exemple, un joueur absorbé dans un suspense ne sera plus en mesure de conserver cette atmosphère dès lors qu'un événement soudain le distraira du manoir lugubre dans lequel il mène une enquête (niveau diégétique) et sera ramené en face de son ordinateur, où il ne sera plus que le témoin d'un ballet de pixels, d'avatars gesticulant sur un écran.

24 L'adjectif « disruptif » prend ici deux significations distinctes. D'une part, il signifie « qui sert à rompre " et désigne les composantes qui, en jeu, sont susceptibles de briser l'organisation d'un cadre et de détourner le joueur d'un niveau de signification. D'autre part, en électricité, cet adjectif se rapporte à un courant électrique qui «transperce un isolant en désorganisant sa structure mécanique et chimique, momentanément ou définitivement » (Académie française, 2005). Un tel phénomène est apte à provoquer un court-circuit, soit la connexion de plusieurs points d'un circuit électrique de tensions différentes (Larousse, 2014). L'analogie illustre ici la capacité d'une figure disruptive à décloisonner des niveaux de signification existant normalement en parallèle les uns les autres, et à les conjoindre dans un même espace. Le concept de figure disruptive permet donc de subsumer les dimensions expérientielle et sociocritique des JRPEV goréens. La première en rendant compte de la rupture des cadres organisateurs de l'expérience de jeu et la seconde, en permettant de décrire comment il y a migration, brouillage ou subversion de socialités concurrentes dans les JRPEV goréens en raison de la conjonction de niveaux de signification normalement séparés les uns des autres, cloisonnés.

Les figures disruptives sont de plusieurs ordres : problèmes techniques (délais de latence et dysfonctions de tous ordres), dispositifs (points de vente automatisés, portails de téléportation, compteurs de points de vie, scripts, etc.), avatars, actions, comportements et répliques de joueurs, règles, accessoires et vêtements des avatars, objets (meubles, véhicules, etc.) et éléments de décors (paysages, édifices, détails architecturaux...), etc.

Le ludique et le diégétique sont les niveaux de signification affectés par une figure disruptive, les niveaux méta-ludique et exégétique (se rapportant à des activités interprétatives) et du hors-jeu (activités non interprétatives) étant les niveaux d'arrivée, une fois la rupture opérée. Nous donnerons plus loin des exemples d'oscillation entre les cadres modalisés et les cadres primaires méta-ludique et du hors-jeu. En ce qui concerne le cadre exégétique, nous mentionnerons les très fréquentes situations au cours desquelles des joueurs BtB interagissent entre eux au niveau diégétique et voient leur expérience perturbée par l'arrivée d'un clan de guerrières venues les attaquer. Cela 
donne typiquement lieu à des échanges d'insultes entre les joueurs, en mode $00 C^{9}$, et à des débats (niveau exégétique) sur le fait que les femmes puissent ou non combattre, selon les informations véhiculées à ce sujet dans les Chroniques. Quant au cadre herméneutique, il est susceptible de se manifester dans le même type de situation, lorsque la question de la femme guerrière devient une réflexion sur la condition féminine dans le monde contemporain ou au cours de l'histoire (Duret, 2013). Toutefois, il serait plus vraisemblable d'envisager qu'une figure disruptive, dans ce contexte, engendre un déplacement entre le niveau diégétique et le niveau exégétique, méta-ludique ou horsjeu, accompagné d'un recadrage herméneutique a posteriori.

Les activités modalisées (le jeu vu aux plans ludique et diégétique) reposent sur ce que Goffman (1991) appelle des conventions de phasage, soit un ensemble de règles socialement partagées ou redéfinies, remises en question dans un contexte social, qui déterminent la manière de les cadrer et d'y prendre part. Identifier ces conventions est crucial pour l'analyste, sans quoi il ne peut situer les interactions des joueurs dans leur contexte respectif. Qui plus est, c'est généralement en raison du non-respect de ces conventions que les figures disruptives entraînent des ruptures de cadre. Toutefois, aux côtés des conventions de phasage, il est également d'autres conventions dont le nonrespect est disruptif : au niveau diégétique, pensons à la cohérence de la diégèse (respect de la logique: un fait, même fictionnel, ne peut être simultanément $\mathrm{A}$ et non- $\mathrm{A}$ ), à sa vraisemblance (on s'attend à ce que des créatures humanoïdes possèdent une tête, qu'une reconstitution de la bataille d'Hastings implique des épées, mais pas des pistolets laser, que personne ne communique à l'aide d'un cellulaire dans un monde de type Steampunk, etc.) et au respect de la thématique de la diégèse (porter des vêtements correspondant à l'époque et à la culture du monde diégétique, adopter ses mœurs et coutumes) et, dans le cas d'une adaptation ou d'une transfictionnalisation, au respect du thème de l'œuvre de référence.

Le fait qu'une figure soit potentiellement disruptive ne signifie pas qu'elle l'est de manière effective. Il est évident qu'elle doit être préalablement perçue par le joueur. Une figure disruptive se caractérise ainsi par une visibilité et une réception variables. Elle peut être invisible tant qu'elle n'est pas activée (c'est le cas d'un script) ou inférée (par exemple, un joueur peut déduire du nombre de points de vie qu'il perd lors d'un combat que l'épée de son adversaire est anormalement et invraisemblablement puissante, ce qui entraînera un échange verbal violent entre son possesseur et lui). Elle peut également n'être accessible qu'à certains avatars, à l'exemple d'un jeu Ouija caché dans un coffre au trésor que seul un voleur saurait ouvrir, ou à certains joueurs : une réplique en slang énoncée par un Goréen est plus ou moins disruptive selon qu'elle est diffusée sur le chat public d'un sim ou par messagerie privée. Ajoutons que les figures disruptives sont de taille variable, de sorte que leur visibilité est, elle aussi, variable. Une reconstitution à l'échelle $1: 1$ d'un zeppelin dans les jardins d'un palais Renaissance est plus visible qu'une autre du Tomato Head de Paul McCarthy. La figure disruptive peut, enfin, être permanente ou temporaire, fixe ou mobile, se présenter fréquemment, à de rares occasions ou une seule fois. Les points de vente d'accessoires goréens sont fixes et permanents, alors qu'une disruption engendrée par un trouble-fête est temporaire et mobile, celle-ci pouvant être unique ou se répéter dans le temps sous la même forme ou sous plusieurs. Dès lors, les probabilités pour qu'un joueur les rencontre diffèrent. Mentionnons que ces dernières caractéristiques impliquent une certaine habitude, de la part du joueur, dans la mesure où il peut $\mathrm{y}$ avoir disruption lors de la ou des première(s) 
rencontre(s) sans que l'effet ne se fasse sentir lors des rencontres subséquentes. Ainsi, un joueur peut trouver disruptive une interface de jeu (objet cyberculturel contemporain) dans un contexte médiéval uniquement lors de ses premières séances et ne plus y prêter attention par la suite, de sorte qu'elle ne perturbe plus son expérience.

Une figure disruptive doit être perçue, mais elle doit également être interprétée pour être effective. La perception est préalable, mais ne va pas sans interprétation, puisque c'est en tant qu'elle est dissonante au regard du contexte dans lequel elle se situe qu'une figure opère une rupture. Cela implique une réception différenciée, soit une lecture plus ou moins fine et exhaustive des implications disruptives de la figure (qui peut constituer une simple rupture de cadre pour l'un et la convocation de significations idéologiques incompatibles pour l'autre, ce qui amène, notamment, la question de l'analyste, par opposition au simple joueur), la mobilisation d'encyclopédies personnelles (Eco, 1985) à étendue et à contenu variables (par exemple, le nom "Trafalgar ", donné à un nain guerrier aquaphobe n'est disruptif que pour les joueurs qui connaissent la bataille éponyme), mais aussi de valeurs (la disruption peut intervenir sous la forme d'une dissonance cognitive) et d'appartenances sous-culturelles variables (certaines expressions émises par un personnage sont disruptives pour un joueur BtB, mais pas pour un GE).

Pour terminer, une figure disruptive affecte l'expérience de manière variable. Il peut $\mathrm{y}$ avoir rupture permanente ou temporaire. Dans le second cas, il est possible de parler de vacillement, soit d'une phase temporaire (et rapide) d'oscillation entre le cadre d'origine et le cadre d'arrivée, puis un retour au cadre d'origine.

\section{Analyse de deux cas de disruption : rupture et vacillement}

31 Nous présenterons maintenant deux cas concrets de figures disruptives: le premier constitue un exemple de vacillement et le second, de rupture de cadre. Le premier exemple a été observé alors que nous explorions, en juin 2012, un village du Torvaldsland, une région nordique de Gor. La figure disruptive est un avatar dont la forme ne respecte pas le thème du sim (la disruption est donc interprétée comme étant inadéquate au regard de la diégèse goréenne). La figure est mobile, temporaire et accessible à tous. Vraisemblablement, elle n'apparaît qu'une seule fois, bien qu'il ne soit pas exclu que l'avatar soit revenu par la suite dans le même sim, occasionnant des perturbations analogues. La taille de la figure disruptive est similaire à celle des autres avatars, si bien qu'il n'est pas fatal que tous le perçoivent: encore faut-il qu'ils croisent sa route, qu'ils apparaissent dans la fenêtre de leur navigateur.

En nous excluant, deux avatars sont présents dans le sim lors de notre visite : l'un est masculin, un guerrier, l'autre féminin, son esclave. Plusieurs indices nous permettent d'affirmer qu'ils se situent au niveau diégétique. Une intrigue amoureuse est construite par le biais de répliques soignées, dans lesquelles des termes appartenant spécifiquement à la culture goréenne sont employés (par exemple, désigner une esclave par le terme goréen « kajira »), par opposition à l'emploi d'expressions argotiques contemporaines et d'un style télégraphique, comme ce serait le cas lors de dialogues énoncés sur le mode OOC. Certaines actions sont décrites verbalement plutôt que performées par les avatars et les dialogues sont rapportés entre guillemets. Ajoutons que l'action est peu importante 
dans leurs interactions, à l'inverse des situations de combat, ce qui exclut la mobilisation du niveau ludique.

Un avatar de raton laveur anthropomorphe fait son entrée, téléporté au milieu de la place publique du village. Lorsqu'elle l'aperçoit, l'esclave hurle d'effroi. Son maître se précipite alors vers l'intrus, l'épée à la main, feignant de vouloir l'abattre. L'esclave est toujours en émoi. Le joueur incarnant le maître, alors que son avatar tourne autour du raton laveur en agitant son arme, écrit alors à ce dernier : «Sors d'ici, ce n'est pas un zoo !». La phrase est inscrite entre doubles parenthèses, ce qui signifie qu'elle est énoncée par le joueur et non son personnage (en OOC). Le raton laveur disparait. Il s'est téléporté hors du village du Torvaldsland. Les personnages poursuivent leur intrigue en y intégrant l'événement étrange qui vient de se produire.

Ici, la figure disruptive est le raton laveur anthropomorphe, un élément qui n'appartient pas à la diégèse goréenne. Il apparaît hors contexte. La disruption a entraîné avec elle un vacillement, dans la mesure où l'intrigue des deux personnages a été temporairement suspendue et l'attention des joueurs s'est portée momentanément sur le raton laveur. Le fait que l'esclave ait hurlé et que son maître se soit porté à sa défense indique qu'ils ont recadré diégétiquement la figure disruptive. Ils ont, en conséquence, rapidement regagné le niveau diégétique. Le maître a ensuite oscillé rapidement entre plusieurs niveaux de signification: il est passé du diégétique au méta-ludique (en expliquant au joueur incarnant le raton laveur que son avatar n'a pas sa place dans un sim goréen et qu'il doit sortir), pour revenir enfin au diégétique.

Le second exemple de figure disruptive témoigne d'une rupture de cadre. Nous la reconstituons à partir du témoignage d'une joueuse, Siobhan Lisle, diffusé dans le forum Gor-SL (2012a). Son personnage discute avec un autre dans une taverne. Tous deux se situent au niveau diégétique lorsqu'ils sont interrompus par deux phrases diffusées sur le chat public du sim où ils se trouvent : la première est énoncée en mode $00 C$ par un joueur, la seconde, par son personnage, un tavernier: «XXX [nom de l'avatar], crie : ((besoin d'une slut maintenant pour servir un visiteur dans la taverne)). XXX crie : tout esclave qui veut servir un homme vient à l'auberge maintenant, s'il-vous-plait ». Dans les deux cas, il s'agit de proposer aux joueurs qui incarnent une esclave (le terme «slut » désignant de manière injurieuse et contemporaine les esclaves de plaisir goréennes) de servir un visiteur masculin qui vient d'entrer dans la taverne. Siobhan Lisle reproche au joueur d'avoir brisé son immersion en ne respectant pas l'étiquette rôliste, c'est-à-dire en émettant une réplique $O O C$ sur un chat public (une réplique que tout le monde est plus ou moins forcé de lire, donc), sans préoccupation pour l'intégrité du niveau diégétique au sein duquel les autres joueurs se situent et en lieu et place de la recherche d'un moyen diégétique de voir combler sa requête. Ici, la perturbation est permanente, la rupture ayant transporté Siobhan Lisle au niveau du hors-jeu. Suite à la disruption, elle se voit ramenée à sa condition de mère qui tente vainement de s'évader de son quotidien, son bébé dans les bras. À ce sujet, elle écrit :

* Siobhan Lisle court dans l'auberge avec mon bébé sur ma hanche pendant que je le nourris au biberon. Il y a du vomi de bébé sur mon épaule parce que je n'ai pas eu le temps de changer mon t-shirt aujourd'hui et je regarde la femme. "as-tu interrompu mon jeu de rôle pour t'y mêler en me criant dessus à travers la subdivision? Parce que tu ne me vois pas? Là, tout de suite, je suis une mère énervée. Je n'ai pas eu le temps de me laver, ma maison est en bazar et j'ai le dîner à préparer bientôt. Fais ton propre foutu jeu de rôle !». 

des formes et des dénominations variables. De manière plus circonscrite, Pierre Zima parle de "situation socio-linguistique ", Yan Hamel, de "mémoire collective ", Claude Duchet de «co-texte » et Philippe Hamon, de « discours idéologiques ». Mais cet ensemble peut être plus vaste et général : c'est le cas de «l'imaginaire social» de Pierre Popovic et du « discours social » de Marc Angenot.

41 La sociocritique distingue le texte et le sociotexte sans exclure le premier aux dépens du second. En effet, écrit Duchet, "[l]e sociotexte n'efface pas le texte des poéticiens [...] Jeter un regard sociocritique c'est le lire comme un sociotexte sans supprimer le fait qu'il est le texte » (in Duchet et Maurus 2011, p. 43), c'est le considérer dans son ouverture à la socialité plutôt que dans son immanence. Ainsi, le «sociotexte est du texte plus du cotexte » (p. 44), du texte en rapport dynamique avec l'ensemble sémiotique socialisé qui l'englobe. 

sans considération pour le rôle accompli par l'expérience vidéoludique dans l'inscription de la socialité au sein des jeux vidéo. C'est que la sociocritique est essentiellement (socio)textualiste. Or, une part de la socialité est charriée par les figures disruptives, dont l'analyse ne peut faire l'économie de la dimension expérientielle du joueur. perturbation de l'expérience vidéoludique. Sans cette perturbation, il n'y a pas conjonction de niveaux de signification ni coprésence d'éléments de socialité potentiellement incompossibles, à l'image d'une diégèse médiévale et de dispositifs techniques (des points de vente automatisés et des portails de téléportation) apportant avec eux une configuration réticulaire et atopique de l'espace, comme nous l'avons vu ailleurs (Duret, 2015b). Qui plus est, en allouant toutes ses ressources attentionnelles au maintien des niveaux diégétique et/ou ludique, le joueur n'est pas en mesure d'activer les potentialités réflexives des éléments de socialité qui lui sont proposés, de recadrer ces derniers au plan herméneutique. En engendrant une rupture de cadre, les figures disruptives libèrent ces ressources attentionnelles et potentialisent des éléments de socialité qui se présentaient auparavant sous une forme latente dans la diégèse et la structure formelle. Ce faisant, elles rendent évidentes la présence d'un sociotexte et mettent en lumière la migration des éléments de socialité entre le hors-jeu et la diégèse, par le biais de la structure formelle et des matériaux sémiotiques (décors, éléments verbaux, images, sons, etc.) du jeu. Sans l'effet de perturbation, les figures disruptives ne s'adressent plus qu'à l'analyste et le jeu perd de sa pertinence critique auprès des joueurs. En prenant en considération la dimension expérientielle des figures disruptives, ce même analyste se voit en mesure de décrire les interactions concrètes du joueur avec la socialité ou, au moins, s'il ne dispose pas des données empiriques nécessaires pour le faire, ses interactions potentielles au sein des champs du possible expérientiels ouverts par ces figures.

Le cas que nous présenterons maintenant a pour but d'illustrer la portée sociocritique des figures disruptives. Comme le second exemple présenté dans la section précédente, il n'est pas tiré de nos propres observations, mais d'un commentaire publié par une joueuse, Elle Couerblanc, dans le forum Gor-SL. Contrairement à Siobhan Lisle, Elle Couerblanc n'est pas une victime de la disruption, mais son initiatrice. Ainsi, elle mentionne plusieurs comportements de trouble-fête qu'elle a adoptés dans plusieurs sims de Second Life, par le biais de ses avatars Lizzie Lexington et Elizabeth Hallstrom, comportements qui ont conduit à son bannissement. Elle écrit :

Bukkake Bliss - Lizzie Lexington bannie pour s'être habillée en avatar DP yumyum bunny ${ }^{10}$ et avoir dansé sur des personnes qui avaient des relations sexuelles en public.

BDSM Ranch - Elizabeth Hallstrom bannie pour avoir dansé au milieu d'un sim avec des chaussures de Pikachu aux pieds.

Plusieurs sims GE l'été dernier - Lizzie Lexington bannie pour s'être à nouveau habillée en avatar DP yumyum bunny et avoir tiré sur des personnes prises au hasard avec un arc (Gor-SL, 2012b).

Ici, les figures disruptives sont l'avatar DP yumyum bunny, les chaussures de Pikachu, la danse et le tir à l'arc de Lizzie Lexington et d'Elizabeth Hallstrom. Les joueurs voient détourné leur engagement aux niveaux diégétique et/ou ludique propres aux sims $G E$, au BDSM Ranch et à Bukkake Bliss par les agissements de Lizzie Lexington et d'Elizabeth Hallstrom. Les joueurs se montrent ainsi dans l'incapacité de maintenir le rôle qu'ils se 
sont assignés dans le cadre de leur jeu de même que le niveau de signification correspondant à ce rôle, soit la fantaisie sexuelle (diégétique ou ludique, selon qu'elle s'inscrive dans une intrigue ou non) dans laquelle ils sont engagés. Ce faisant, ils délaissent involontairement le niveau ludique ou diégétique pour celui du hors-jeu ou du méta-ludique.

Ce qui nous intéresse ici, c'est la portée sociocritique des disruptions décrites par Elle Couerblanc. En tant que figures disruptives, l'avatar DP yumyum bunny et les chaussures de Pikachu favorisent la rencontre en un même espace et dans un même médium de diégèses normalement étrangères les unes aux autres. Cette rencontre est possible en raison de leur valeur synecdochique, la partie, soit DP yumyum bunny et Pikachu, valant pour le tout, c'est-à-dire l'univers au sein duquel ces personnages évoluent respectivement. De telles figures mettent alors en scène, sur les modes de la parodie et de l'antithèse (le bukkake et la sexualité BDSM constituant des pratiques situées à l'opposé du monde de l'enfance auxquels Pikachu et DP yumyum bunny appartiennent), ce qu'Henry Jenkins (2006) qualifie de convergence culturelle, phénomène né de la rencontre de la convergence médiatique et de la culture participative.

Selon Jenkins, la convergence médiatique se rapporte à la circulation des flux de contenus à travers de multiples plateformes médiatiques, à la coopération entre de multiples industries médiatiques, au comportement migratoire de l'audience des médias qui va en tout lieu à la recherche du type de divertissement qu'il désire. La convergence décrit à la fois les changements technologiques, industriels, culturels et sociaux. La culture participative, quant à elle, et toujours selon Jenkins, est une «culture dans laquelle les fans et les consommateurs sont invités à participer activement à la création et à la circulation de nouveaux contenus " (Jenkins, cité dans François, 2009, p. 217). Fans et consommateurs de médias sont vus comme des récepteurs actifs. Ils s'approprient les médias en participant à la production, au mixage, à l'extension et à la transformation amateurs de contenus audiovisuels, vidéoludiques, photographiques et littéraires.

C'est donc un mode de production et de consommation médiatique représentatif de la convergence culturelle qui est convoqué par les figures disruptives décrites. Ces dernières constituent une initiative amateur de mise en coprésence de diégèses antithétiques dans un MUVE ouvert, Second Life, au sein duquel les usagers se voient en mesure de concevoir les décors, les scripts, les dispositifs techniques, les objets et les règles du jeu (culture participative). Le MUVE en question devient l'une des nombreuses plateformes, avec les animes, les jeux de cartes à collectionner, les mangas, les jeux vidéo, etc., sur lesquelles circulent un personnage tel que Pikachu (convergence médiatique). Sa présence n'a pas pour objectif de tisser un récit transmédiatique dans le cadre d'une franchise commerciale. Il a pour but d'agir à titre de trouble-fête et d'interrompre l'expérience diététique et/ou ludique des usagers sur les sims GE, dans BDSM Ranch et Bukkake Bliss Island.

\section{Conclusion}

Aussi exotiques soient les univers vidéoludiques proposés, des galaxies lointaines de Star Wars aux contrées pseudo-médiévales de Lord of the Rings, tous sont émaillés d'éléments de socialité témoignant de leur porosité vis-à-vis du monde contemporain, de sorte qu'il y a lieu de qualifier les jeux vidéo d'objets aux frontières osmotiques, par opposition à une vision formaliste qui en fait des objets aux frontières hermétiques. Nombre de ces 
éléments s'inscrivent dans la diégèse lorsqu'aucun rapiéçage métaphorique (Stern, 2002) ne vient dissimuler les composantes de la structure formelle ou lorsqu'aucun recadrage d'objets, de personnages ou de situations de nature disruptive n'est effectué en termes diégétiques. Il convient à la sociocritique des jeux vidéo de rendre compte de ces situations de coprésence d'éléments de socialité incompatibles et incompossibles convoqués par les figures disruptives tout en tenant compte des caractéristiques de l'expérience vidéoludique qui les suscitent. C'est grâce à cette attention que peut être mise en lumière la portée critique des jeux vidéo, sa capacité à faire communiquer entre eux et à transformer en les confrontant une multitude de points de vue exprimés en un chant polyphonique discordant.

\section{BIBLIOGRAPHIE}

Académie française (2005), « Disruptif », Dictionnaire de l'Académie française, $9^{\mathrm{e}}$ éd. Tome 1, A-Enz, Paris, Fayard.

BATESON G. (1977), « Une théorie du jeu et du fantasme », Vers une écologie de l'esprit, Tome I, Paris, Éditions du Seuil.

CALLEJA G. (2011), In-Game. From Immersion to Incorporation, Cambridge, The MIT Press.

CASSAR R. (2013), « Gramsci and Games », Games and Culture, 8 (5), pp. 330-353.

CRIST. (n.d.), « Manifeste », http://www.site.sociocritique-crist.org/ p/manifeste.html

CROGAN P. (2011), Gameplay mode. War, simulation, and technoculture, Minneapolis, University of Minnesota Press.

DORMANS J. (2006), « On the role of the die. A brief ludologic study of pen-and-paper roleplaying games and their rules, Game studies, 6 (1), http://gamestudies.org/0601/articles/dormans

DUCHET C. \& MAURUS P. (2011), Un cheminement vagabond. Nouveaux entretiens sur la sociocritique, Paris, Honoré Champion.

DURET C. (2013), « Jeux de rôle participatifs en environnement virtuel et communauté herméneutique conflictuelle. L'expérience ludique des joueurs sur les "sims" goréens », Communication, lettres et sciences du langage, 7 (1), pp. 4-20.

DURET C. (2014a), « Les cadres de l'expérience vidéoludique et la distribution des ressources attentionnelles dans les jeux de rôle en ligne. Une alternative à la notion d'immersion », Revista Contracampo, 29 (1), pp. 84-108.

DURET C. (2014b), « Living the Phantasm of Demediation. The Priest Kings and the Technology Prohibition in the Gorean Role-Playing Games », Religion in Digital Games Reloaded, Heidelberg Journal of Religions on the Internet, 7, pp. 41-60.

DURET C. (2015a, à paraître), « Entre hétérotopies et non-lieux. Le brouillage et la subversion du fantasme de démédiation dans les jeux de rôle goréens », in Menassel M. et Pierquin E. (dir.), Espace perçu, espace vécu, espace rêvé, Valenciennes, Presses universitaires de Valenciennes. 
DURET C. (2015b, à paraître), « Non-médiation, démédiation et surmédiation. $\tilde{\eta} \theta$ o et éthos dans les jeux de rôle goréens », Ethos numériques, Itinéraires. Littérature, textes, cultures.

DURET C. (à paraître), « Penser les frontières des jeux de rôle participatifs en environnement virtuel : Le cas des jeux de rôle goréens de Second Life ».

DYER-WITHEFORD N. \& DE PEUTER G. (2009), Games of Empire. Global Capitalism and Video Games, Minneapolis, University of Minnesota Press.

ECO U. (1985), Lector in fabula, le rôle du lecteur ou la Coopération interprétative dans les textes narratifs, Paris, Éditions Grasset et Fasquelle.

FINE G. A. (1983), Shared Fantasy. Role-Playing Games as Social Worlds, Chicago, Londres, University of Chicago Press.

FRANÇOIS S. (2009), « La participation médiatique selon Henry Jenkins (note critique) », Terrains \& travaux, 15, pp. 213-224.

GADE M. (2003), « Interaction. The key element of larp », in Gade M., Thorup L. et Sander M. (dir.), As LARP Grows Up. The Book from Knudepunkt, Frederiksberg, Projektgruppen KP03, pp. 66-71. GOFFMAN E. (1991), Les cadres de l'expérience, Paris, Éditions de Minuit.

Gor-SL. (2012a), « Don't Ruin My Immersion By Breaking Yours! », http://www.gor-sl.com/ index.php/topic,12335.msg107097.html\#msg107097

Gor-SL. (2012b), » Sim Bans out of hand? », http://www.gor-sl.com/index.php/ topic, $12644.60 . \mathrm{html}$

HIGGIN T. (2009), «Blackless fantasy. The disappearance of race in massively multiplayer online role-playing games », Games and Culture, 4 (1), pp. 3-26.

HITCHENS M. \& DRACHEN A. (2009), « The Many Faces of Role-Playing Games », International Journal of Role-Playing, 1 (1), pp. 3-21.

HOLTER M. (2007), « Stop saying “immersion”! », in Donnis J., Gade M. et Thorup L. (dir.), Lifelike, Copenhague, Projektgruppen KP07, Landforeningen for Levende Rollespil, pp. 20-22.

JENKINS H. (2006), Convergence Culture: Where Old and New Media Collide, New York et Londres, New York University Press.

JUUL J. (2010), A Casual revolution. Reinventing Video Games and Their Players,

Cambridge, The MIT Press.

LANGER J. (2008), « The Familiar and the Foreign. Playing (Post)Colonialism in World of Warcraft », in H. G. Corneliussen et J. W. Rettberg (dir.), Digital culture, play, and identity: a World of Warcraft Reader, Cambridge, The MIT Press, pp. 87-108.

Larousse (2014), « Court-circuit, courts-circuits », http://www.larousse.fr/dictionnaires/ francais/court-circuit_courts-circuits/19961

LETSON R. (1978), « Introduction », in P. J. Farmer, Green Odyssey, Boston, Gregg Press, 152 p.

LIZARDI R. (2009), « Repelling the Invasion of the "Other". Post-Apocalyptic Alien Shooter Videogames Addressing Contemporary Cultural Attitudes », Eludamos, 3 (2), pp. 295-308.

MASSIE K. (2011), « Representations of Race and Gender within the Gamespace of the MMO "Everquest" », in G. Crawford,V. K. Gosling et B. Light (dir.), Online Gaming in Context. The Social and Cultural Significance of Online Games, Abingdon, Routledge. 
MAUCO O. (2013), GTA IV, l'envers du rêve américain. Jeux vidéo et critique sociale, Paris, Questions Théoriques.

McCAULEY D. (2008), « Power up. Do PCs stand a chance against consoles? Maybe », The Philadelphia Inquirer (18 avril 2008).

MONSON M. J. (2012), "Race-based fantasy realm. Essentialism in the World of Warcraft ", Games and Culture, 7, pp. 48-71.

MORGAN C. D. (2002), « The alchemy of role-playing », http://indie-rpgs.com/archive/ index.php?topic=5608.0; wap2

PADOL L. (1996), « Playing stories, telling games. Collaborative storytelling in role-playing games ", www.labcats.org/drcpunk/wiki/index.php/Playing_Stories,_Telling_Games

PECK B. M., KETCHUM P.R. \& EMBRICK D.G. (2011), « Racism and sexism in the gaming world: Reinforcing or changing stereotypes in computer games? ", Journal of Media and Communication Studies, 3 (6), pp. 212-220.

PINEL K. (2014), « Machinarium. Du plaisir esthétique postmoderne », Interfaces numériques, 3 (1), pp. 101-113.

Reuter (2012), « Factbox. A look at the $\$ 78$ billion video games industry », http:// www.reuters.com/article/2012/06/01/us-videogameshow-e3-show-factboxidUSBRE8501IN20120601

RICOEUR P. (1986), Du texte à l'action. Essais d'herméneutique II, Paris, Éditions du Seuil.

SIANG ANG C., ZAPHIRIS P. \& WILSON S. (2010), « Computer Games and Sociocultural Play: An Activity Theoretical Perspective », Games and Culture, 4 (5), pp. 354-380.

SMITH D. A. (1996), « No More Gor. A Conversation with John Norman: part one », The New York Review of Science Fiction, 8 (92), document non paginé.

STENROS J. \& HAKKARAINEN H. (2003), « The Meilahti school thoughts on role-playing », in Gade M., Thorup L. et Sander M. (dir.), As LARP Grows Up. The Book from Knudepunkt, Frederiksberg, Projektgruppen KP03, pp. 54-64.

STERN E. (2002), « A touch of medieval. Narrative, magic and computer technology in massively multiplayer computer role-playing games ", in Mayra F. (dir.), Computer Games and Digital Cultures Conference Proceedings. Tampere, Tampere University Press.

THON J.-N. (2008), « Immersion Revisited. On the Value of a Contested Concept », Extending Experiences, in Olli L., Wirman H. et Fernandez A. (dir.), Structure, Analysis and Design of Computer Game Player Experience, Rovaniemi, Lapland University Press, pp. 29-43.

\section{NOTES}

1. Selon la perspective axée sur l'objet (game-centric view), le jeu dicterait les actions que le joueur est susceptible d'y poser, alors que selon la perspective axée sur le joueur (player-centric view), tout ce qui advient dans le cadre du jeu relèverait plutôt du joueur.

2. Dans Second Life, les scripts sont des lignes de programmation qui prennent la forme d'objets qu'un usager peut conserver dans son inventaire. Ils permettent d'obtenir certains effets : modifier l'apparence d'un objet, comment il se déplace, ses interactions et ses échanges avec des avatars, etc. 
3. La romance planétaire constitue un sous-genre de la science-fiction, dont les œuvres font le récit d'aventures se déroulant sur une planète autre que la Terre. Le récit est intimement relié à la description de cette planète. Les romances planétaires se distinguent des opéras spatiaux dont Star Wars et Star Trek sont des exemples connus -, mais aussi de la fantasy, en raison du fait qu'elles se déroulent dans le cadre d'une planète exotique et technologiquement primitive, malgré qu'elles partagent avec eux les conventions des récits d'aventure telles que les poursuites, les évasions et les quêtes (Letson, 1978).

4. Nous reprenons ici, dans une version synthétisée, la section « Une tripartition des JRPEV en ses niveaux de signification constitutifs", tirée de notre chapitre «Penser les frontières des jeux de rôle participatifs en environnement virtuel : Le cas des jeux de rôle goréens de Second Life » (en cours d'évaluation).

5. Récits relatant après coup les événements survenus en situation de jeu.

6. Les sims, aussi appelés "simulateurs", sont des espaces virtuels hébergés sur des serveurs loués par Second Life à ses usagers, qui les aménagent et les administrent selon leur bon vouloir.

7. Par exemple, vaincre l'ennemi, faire preuve de précision et d'agilité dans la manipulation de l'avatar, accumuler des richesses ou des points, remplir l'ensemble des conditions menant à la victoire et le faire le plus rapidement possible ou en ne perdant aucun point de vie sont typiques d'actions ludiques.

8. Dans les jeux en ligne et les MUVE, le trouble-fête, ou griefer, est un individu qui adopte des comportements jugés inappropriés (harcèlement, insultes, actions entraînant des dysfonctions au sein du jeu ou de l'environnement virtuel) par les joueurs/usagers qui en sont victimes.

9. $00 C$ (Out of Character) : situation où les actions et les répliques du joueur sont attribuables à luimême et non à son personnage, c'est-à-dire lorsque ce dernier délaisse temporairement son rôle. 10. Avatar de lapin anthropomorphe.

\section{RÉSUMÉS}

Cet article porte sur les figures disruptives présentes dans les jeux de rôle goréens organisés dans Second Life. Elles sont vues comme des formes d'intrusion et de contamination de l'expérience vidéoludique des joueurs, provoquant l'oscillation de ces derniers entre les différents cadres et niveaux de signification qui en sont constitutifs. Si les figures disruptives revêtent une dimension expérientielle évidente, nous verrons qu'elles sont également pourvues d'une portée critique lorsqu'elles charrient avec elles des éléments de socialité incompatibles avec la diégèse du jeu, en provenance, notamment, de la structure formelle du jeu ou en conséquence de la violation des règles ou de l'étiquette du jeu de rôle.

This article focuses on disruptive figures present in Gorean role-playing games organized in Second Life. They are seen as forms of intrusion and contamination of the gaming experience of the players, causing oscillation between different frames and levels of meaning that are constitutive of this experience. If the disruptive figures are of obvious experiential dimension, we will see that they also have a critical impact when they carry with them sociality elements incompatible with the diegesis of the game. These elements come from the formal structure, but they also are consequences of the violation of rules or etiquettes of role play. 
INDEX

Mots-clés : environnements virtuels multi-usagers, jeux de rôle, jeux de rôle en ligne, jeux de rôle participatifs en environnement virtuel, JRPEV, Second Life, sociocritique

Keywords : online multi-user virtual environments, online role-playing games, participatory role-playing games in virtual environment, PRPG-VEs, sociocriticism

\section{AUTEUR}

\section{CHRISTOPHE DURET}

Université de Sherbrooke 